\title{
New type of the source of travelling impulses in two-variable model of reaction-diffusion system
}

\author{
Andrzej L. Kawczyński ${ }^{1}$ Bogdan Nowakowski ${ }^{1,2}$
}

Received: 27 November 2015/ Accepted: 12 February 2016/Published online: 24 February 2016

(C) The Author(s) 2016. This article is published with open access at Springerlink.com

\begin{abstract}
A two-variable model of a one-dimensional, open, excitable, finite reaction-diffusion system describing time-space evolution of traveling impulses is investigated. It is shown that depending on the size of the system, the traveling impulse after reflection can generate either a source of decaying traveling impulses or a stationary periodical structure. A continuous increase of the size of the system causes periodical repetitions of these patterns. The chemical model is realistic and can become a stimulus for seeking the described effect in experiments.
\end{abstract}

Keywords Excitable reaction-diffusion system - Spatially periodic chemical structure $\cdot$ Chemical traveling wave $\cdot$ Enzymatic reaction

\section{Introduction}

Among a variety nonlinear effects observed in far from equilibrium chemical systems [1-15], the best known and the easiest to demonstrate is the target pattern discovered in the Belousov-Zhabotinsky (BZ) reaction with ferroine as the catalyst. 45 years ago Zaikin and Zhabotinsky had announced their discovery of the phenomenon which they called a leading center [16]. In an unstirred orangered thin layer (1-2 mm) of homogenously oscillating or excitable mixture of $\mathrm{KBrO}_{3}$, bromomalonic acid, ferroine and sulfuric acid, small regions (sources of impulses, generators of impulses) appear spontaneously from which many running waves in the form of blue rings propagate. Now this phenomenon (or its movie) is

Bogdan Nowakowski

bnowakowski@ichf.edu.pl

1 Institute of Physical Chemistry, Polish Academy of Sciences, Kasprzaka 44/52,

01-224 Warsaw, Poland

2 Physics Laboratory, Warsaw University of Life Sciences SGGW, Nowoursynowska 159, 02-776 Warsaw, Poland 
often demonstrated as the example of the self-organization in far from equilibrium chemical systems. It is evident that the frequency of oscillations in the sources of impulses is higher than in the surrounding medium. There was the problem of a theoretical explanation of the target patterns in the BZ system, but their discovery put the general question. What is the reason of the increase of the frequency of oscillations or the local switch of the system from excitable to oscillatory regime in reaction-diffusion systems? The simplest answer to this problem is a pacemaker concept $[17,18]$. A local heterogeneity (e.g. unidentified impurity) by definition increases the frequency of oscillations in the region from which many impulses of excitations propagate. Consequently, for models based on the pacemaker concept, the reaction-diffusion equations have to include some specific terms which depend on the space coordinate in an explicit way [17, 18]. Because oscillations of reagent concentrations can appear in two-variable models, the general model for target patterns caused by pacemakers consists of two reactiondiffusion equations $[17,18]$. Using the pacemaker concept, Field and Noyes were able to explain the target patterns on the base of the Field, Körös and Noyes (FKN) mechanism of the BZ reaction [19]. The pacemaker can induce the periodically spreading impulses also in an excitable regime of any reactiondiffusion system. The pacemaker concept introduces two different reaction dynamics in the reaction-diffusion systems. The chemical dynamics in the region which generates the traveling impulses exhibits higher frequency than the chemical dynamics in the surrounding medium.

However, there is the other possibility of the explanation of the target pattern phenomenon in reaction-diffusion systems based on the leading center concept. This is a more intriguing possibility, because it points to a spontaneous process of self-organization in reaction-diffusion systems. Namely, sufficiently large fluctuations appear in some place of the oscillatory reaction-diffusion system, which locally overtake a phase of oscillations towards the blue state. This shift in the phase of oscillation changes the reaction dynamics in such a way that a frequency of oscillations locally increases and this increment of the frequency becomes persistent. The corresponding reaction-diffusion equations cannot depend on space coordinates in an explicit way. The leading center (source of traveling impulses, generator of traveling impulses) should be generated due to the chemical dynamics which is identical in the whole system. The general problem was to construct a simple model describing the target pattern formation in the system consisting of a minimal number of reaction-diffusion equations. Therefore, the first models of the leading center were abstract ones. They were based on activator-inhibitor types mechanisms [20, 21]. The model constructed by Zhabotinsky and Zaikin [20] consisted of two reaction-diffusion equations of the activator-inhibitor type but the cause of generation of the leading center was difficult to explain. In the next abstract model describing the appearance of a target pattern in excitable system of the activator-inhibitor type, one variable was added in order to shift a position of nullcline of the inhibitor from excitable to the oscillatory regime [21].

Because the mechanism of the BZ reaction is very complex in the sense that the dynamics of this reaction is described by many-variable models, it is difficult to prove that the target patterns observed in this system are generated by leading 
centers. To our best knowledge, it is still an open problem. There is no doubt that the target patterns can be generated by reproducible voltage pulses across two electrodes (playing the role of the pacemaker) dipped in the reaction mixture [19]. The more realistic three-variable models of the leading center were based on enzymatic (catalytic) reactions with the quadratic activation by an excess of a product with additional variable shifting the position of the nullcline for the inhibitor from the excitable to the oscillatory regime in the region of initial excitation [22]. The model of so called non-sustained leading center based on enzymatic reactions had been constructed [23, 24]. In this model, an initial excitation of the system generates a given, finite number of traveling impulses spreading as a packet. After some refractory time, the next excitation in the same place of the system generates the same number of traveling impulses and so on. The above models consist of at least three reaction-diffusion equations, two of them are the activator-inhibitor type in excitable regime. It appears that the leading center (source of traveling impulses, generator of traveling impulses) can appear as the result of initial local excitation in two-variable reaction-diffusion systems in the bistable regime when a stable focus is separated from a stable limit cycle by an unstable limit cycle [25, 26]. The traveling impulses created in the model [26] have unusual form. They have a local minimum between their fronts and backs.

In the present paper, another possibility is shown, which, to our best knowledge, has not been described so far. It is known that a propagating impulse can be reflected from an impermeable boundary, and subsequently can travel bouncing from walls limiting a one-dimensional (1D) system [27, 28]. We have found that after reflection from the impermeable boundary, a traveling impulse can periodically generate infinite number of traveling impulses spreading towards the opposite boundary and decaying just before it. This new type of leading center coexists with the spatially periodic stationary structure for other initial conditions. The stationary structure appears when the traveling impulse after reflection from the impermeable boundary reflects also on the opposite boundary. The new type of the source of traveling impulses (leading center) appears only in some sizes of the system. This evolution is repeated periodically with the continuous increase of the size of the system. Such a periodical dependence appears in the finite excitable system described by a realistic chemical model. The considered model has three stationary states. One of them is a stable node and the two others are a saddle point and an unstable focus. The same model has been used previously for the generation of large amplitude spatially periodic stationary structures created by subsequent divisions of a traveling impulse [29]. Moreover, two-dimensional (2D) stationary patterns mimicking all capital Latin [30] as well as Old Hebrew [31] letters have been generated in the same model. We have also shown that an absolutely stable traveling impulse in a $1 \mathrm{D}$ system becomes unstable in a $2 \mathrm{D}$ system provided that the size of the system perpendicular to the impulse propagation is sufficiently large [32].

In the next chapter, we describe the model and the results of numerical calculations. The last chapter presents the conclusions. 


\section{Model and results}

The model describes an open chemical system in which two catalytic (enzymatic) reactions occur.

$$
\begin{aligned}
& k_{1} \\
& S_{0} \Leftrightarrow S \\
& k_{-1} \\
& k_{2} \\
& S+E \Leftrightarrow S E \\
& k_{-2} \\
& S E \stackrel{k_{3}}{\longrightarrow} P+E \\
& k_{4} \\
& S+S E \Leftrightarrow S_{2} E \\
& k_{-4} \\
& k_{5} \\
& P+E \Leftrightarrow E P \\
& k_{-5} \\
& k_{5} \\
& P+S E \Leftrightarrow S E P \\
& k_{-5} \\
& k_{5} \\
& P+S_{2} E \Leftrightarrow S_{2} E P \\
& k_{-5} \\
& k_{6} \\
& P+E^{\prime} \Leftrightarrow P E^{\prime} \\
& k_{-6} \\
& P E^{\prime} \stackrel{k_{7}}{\longrightarrow} R+E^{\prime} \\
& P \stackrel{k_{8}}{\longrightarrow} Q
\end{aligned}
$$

The core of the model is the Michaelis-Menten mechanism of enzymatic reaction (Eqs. 2 and 3), which transforms substrate $S$ into enzymatic product $P$. An excess of the substrate $S$ (activator) and the product $P$ (inhibitor) inhibits the production of $P$ (Eqs. 4-7). The Michaelis-Menten scheme with inhibition steps describes correctly kinetics of many single-substrate enzymes, like carboxylesterase and isomerase families. For simplicity, we assume that the other catalytic reactions (Eqs. 8-9) occur in its saturation regime. The system is open due to reaction 1 , in which $S_{O}$ plays the role of the reservoir variable for the reactant $S$, and reaction 10 , 
in which the product $P$ is irreversibly transformed to inactive reagent $Q$. It is noteworthy that the chemical scheme consists of elementary reactions, that is monomolecular and bi-molecular reactions without autocatalytic steps.

We assume that the total concentrations of catalysts (enzymes) $E$ and $E^{\prime}$ are much smaller than the concentrations of the reactant $S$ and the product $P$. On the basis of the Tikhonov theorem [33], the concentrations of both catalysts (enzymes) and their complexes may be eliminated as fast variables, and the dynamics of the system may be described by the two kinetic equations for the reactant $S$ and the product $P$ only.

The time-space behavior of the system for dimensionless concentrations of the reactant $s$ and the product $p$ is described by the following equations:

$$
\begin{gathered}
\frac{\partial s}{\partial t}-\frac{\partial^{2} s}{\partial x^{2}}=A_{1}-A_{2} s-\frac{s}{\left(1+s+A_{3} s^{2}\right)(1+p)} \\
\frac{\partial p}{\partial t}-D \frac{\partial^{2} p}{\partial x^{2}}=B\left(-B_{1}-B_{2} p+\frac{s}{\left(1+s+A_{3} s^{2}\right)(1+p)}\right)
\end{gathered}
$$

Here $s=S / K_{m}$ and $p=K_{5} P$ are dimensionless concentrations of the reactant $S$ and the product $P$, respectively, $D=D_{p} / D_{s}$ is the ratio of the diffusion coefficients for the product $D_{p}$ and the reactant $D_{s}, x=\left(k_{3} E_{0} / D_{s} K_{m}\right)^{1 / 2} x^{\prime}$ is the dimensionless space coordinate, $t=k_{3} E_{0} / K_{m} t$ ' is dimensionless time, $K_{5}=k_{5} / k_{-5}$ is the equilibrium constant, and $K_{m}=\left(k_{-2}+k_{3}\right) / k_{2}, K_{m^{\prime}}=\left(k_{-6}+k_{7}\right) / k_{6}$ are the Michaelis constants. $A_{1}=k_{1} S_{0} /\left(k_{3} E_{0}\right), \quad A_{2}=k_{-1} K_{m} /\left(k_{3} E_{0}\right), \quad A_{3}=k_{4} / k_{-4} K_{m}$, $B=K_{m} K_{5}, B_{1}=k_{7} E_{0^{\prime}} /\left(k_{3} E_{0}\right)$ and $B_{2}=k_{8} /\left(k_{3} E_{0} K_{5}\right)$ are dimensionless parameters. The assumption that reactions 8 and 9 occur in its saturation regime means that $K_{m^{\prime}}$ is much smaller than $p$. Therefore, the rate of this reaction is constant and equal to $B_{1}$.

Let us mention that the parameters $B$ and $D$ do not have any influence on stationary states nor on the nullclines of the system 11 and 12 . Therefore, $B$ and $D$ can be treated as bifurcation parameters. Choosing the values of the remaining parameters, our intention is to obtain the monostable, excitable dynamics of Eqs. 11 and 12, which can give propagating impulses in a spatially extended 1D system [2932]. In reference to real systems, it should be noticed that reaction rate constants can vary in a very wide range, even if values of diffusion coefficients of simple molecules in liquids are not very widely dispersed $\left(\sim 10^{-5} \mathrm{~cm}^{2} / \mathrm{s}\right.$ at ambient temperature). Thus, it is possible to match the values of rate constants in the combinations defining the above parameters to obtain experimentally feasible scales. The following values of the parameters are assumed:

$$
A_{1}=10^{-2}, A_{2}=10^{-4}, A_{3}=0.505, B_{1}=7.99 \times 10^{-3}, B_{2}=4.65 \times 10^{-5}
$$

The nullclines for the activator $s$ and the inhibitor $p$ for the above values of the parameters have the following forms, respectively: 


$$
\begin{gathered}
p=\frac{s}{\left(1+s+A_{3} s^{2}\right)\left(A_{1}-A_{2} s\right)}-1 \\
p=\frac{1}{2}\left[\left(\left(B_{12}+1\right)^{2}+4\left[\frac{s}{B_{2}\left(1+s+A_{3} s^{2}\right)}-B_{12}\right]\right)^{1 / 2}-\left(B_{12}+1\right)\right]
\end{gathered}
$$

In Eq. 14, we denote $B_{12}=B_{1} / B_{2}$. The graphs of the nullclines are shown in Fig. 1. Only a small part of the upper attracting branch of the nullcline for $s$ is shown. For assumed values of the parameters, system 11 and 12 has three stationary states: the stable node $(S N)$, the saddle point $(S P)$ and the third stationary state, whose stability depends on $B$. In the sequel, we assume that $0.418 \leq B \leq 0.422$. In this range of $B$, the third stationary state is unstable focus $(U F)$. The well-separated unstable focus is visible in Fig. 1. The insert in Fig. 1 enlarges the region where the nullclines are very close and have two additional intersection points: $S N$, and $S P$ located between $S N$ and $U F$ along the nullclines.

The initial-boundary value (Fourier) problem for the system in Eqs. 11 and 12 on the space interval $[0, \mathrm{~L}]$ for initial values: $s(0, x)=20, p(0, x)=35$ for $0 \leq x<0.5$, and $s(0, x)=1.22754, p(0, x)=40.5859$ for $0.5 \leq x \leq L$, and boundary conditions $\left.\frac{\partial s}{\partial x}\right|_{0}=\left.\frac{\partial s}{\partial x}\right|_{L}=\left.\frac{\partial p}{\partial x}\right|_{0}=\left.\frac{\partial p}{\partial x}\right|_{L}=0$ is solved numerically. The initial values of $s$ and $p$ on the unexcited interval $[0.5, \mathrm{~L}]$ are approximately equal to their values at the stable node. We solve Eqs. 11 and 12 numerically using the Cranck-Nicholson scheme for the diffusion terms, and the fourth order Runge-Kutta algorithm for the kinetic terms. In order to avoid numerical artifacts, we have changed (decreasing and increasing) the spatial step in the range from 0.0025 to 0.01 and the time step in

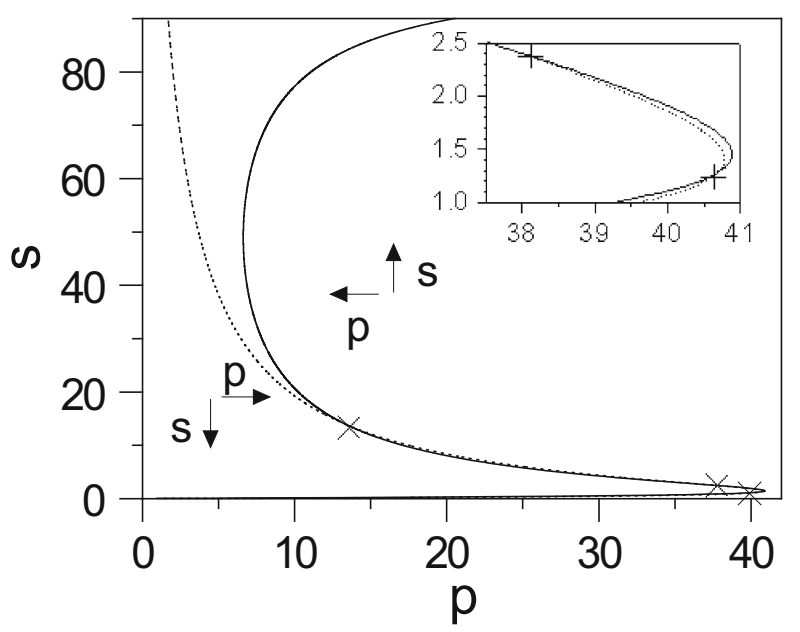

Fig. 1 The nullclines for $s$ (continuous line) and $p$ (dotted line) on the phase plane ( $p$,s) for Eqs. 11 and 12 with neglected diffusion terms. The unstable focus $(U F)$ positioned at $(13.36431468,13.88559325)$ is visible. The inset shows that the nullclines have two additional intersection points: the stable node $(S N)$ with coordinates $(40.5859278,1.22754247)$ and the saddle point $(S P)$ at $(38.144272305,2.362912366)$ 
the range from 0.5 to 2 . The results of numerical calculations presented below do not depend on the used spatial and time steps in the ranges given above.

Examples of space-time evolutions of initial excitation of Eqs. 11 and 12 with $B=0.42$ and $D=3.065$ are shown in Figs. 2, 3 and 4 for three systems with $L=21.0$ and $L=21.2$ and $L=21.435$. Before reflection from the right boundary, a traveling impulse has an invariable front and slightly oscillating back. After reflection, the traveling impulse splits into a new traveling impulse and an excited pulse near the right boundary. The traveling impulse spreading to the left boundary leaves behind periodically decaying pulses. The excited pulse near the right boundary remains in the excited state. Next the excited region forming the pulse splits again forming a subsequent traveling impulse. Further evolution depends on the size of the system. For the system shown in Fig. 2, a traveling impulse reflects from the left boundary and asymptotically generates a spatially periodic stationary structure. However, if the size of the system is changed a little, then the region of splitting may periodically create traveling impulses decaying before the left boundary, creating a specific source of decaying impulses (see Fig. 3). Further increasing of the system size causes a traveling impulse again to reflect from the left boundary and asymptotically creates a spatially periodic stationary structure (see Fig. 4). The only difference between the systems shown in Figs. 2, 3 and 4 is that they have different sizes.

The number of decaying pulses left behind during the spreading of the traveling impulse towards the left boundary increases by one if the size of the system grows

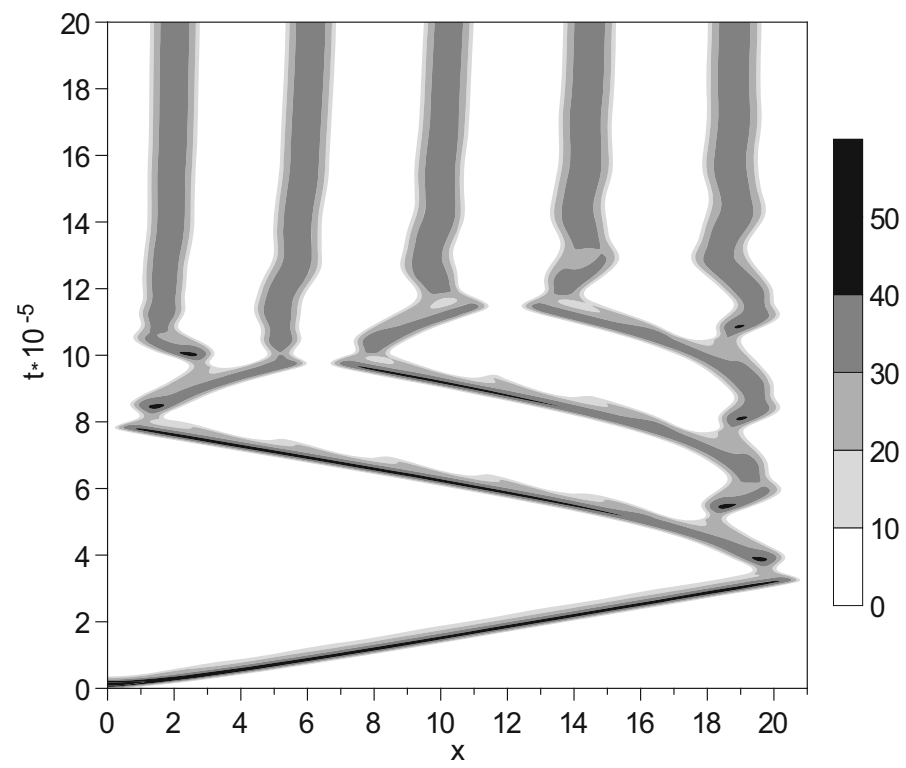

Fig. 2 Time-space evolution of $s(t, x)$ (activator) in the system for $B=0.42$ and $D=3.065$ and $L=21.0$. At this size the traveling impulse after reflection from the right boundary generates a sustained source of decaying traveling impulses 


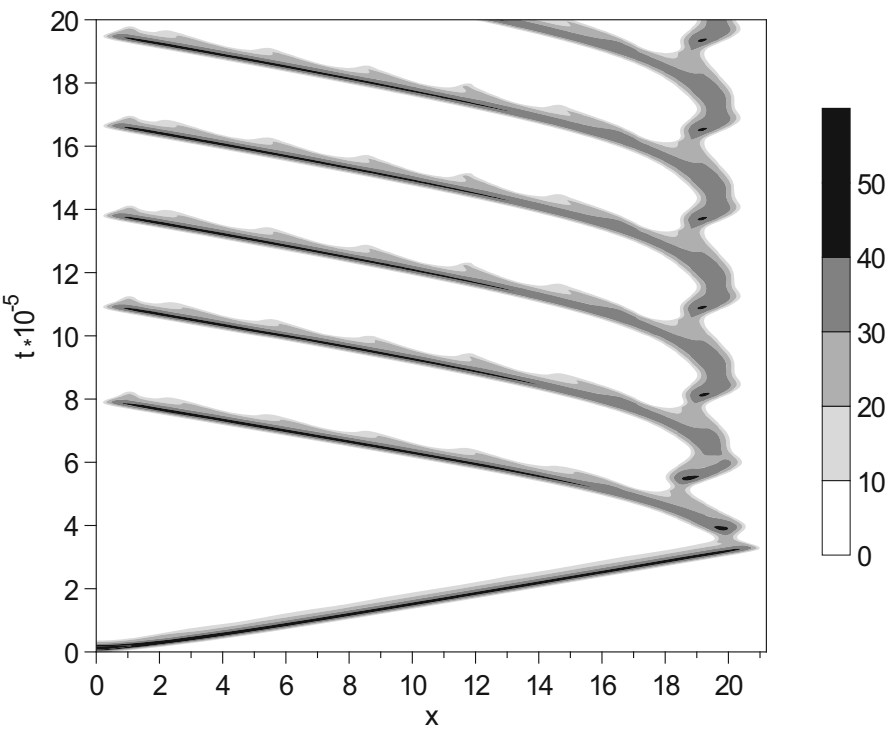

Fig. 3 Time-space evolution of $s(t, x)$ (activator) in the system for $B=0.42$ and $D=3.065$ and $L=21.2$. At this size the traveling impulse decays before the left boundary and a sustained source of decaying impulses appears

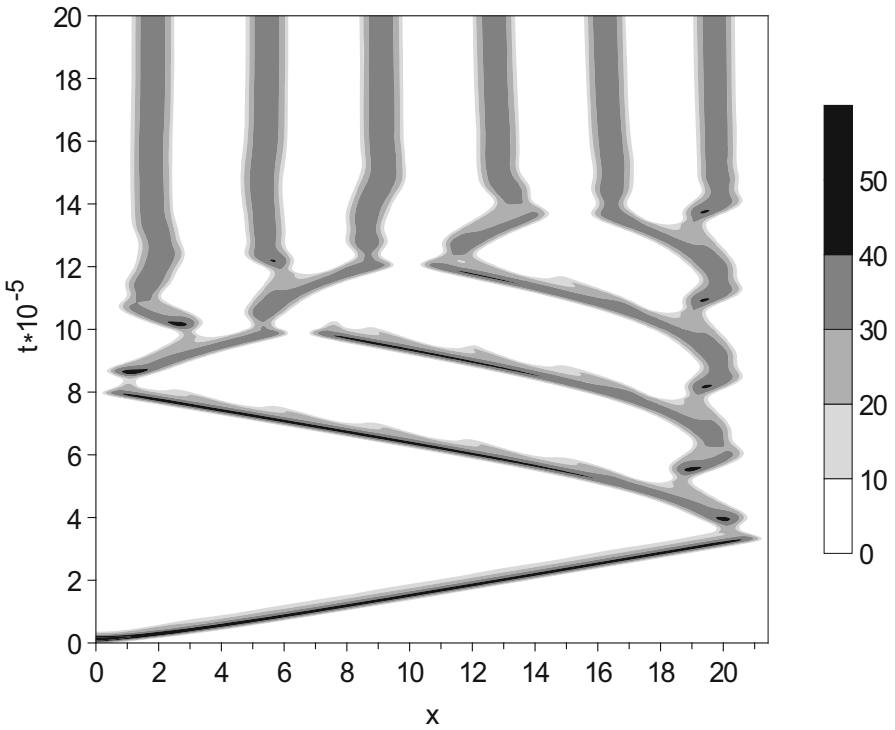

Fig. 4 Time-space evolution of $s(t, x)$ (activator) in the system for $B=0.42$ and $D=3.065$ and $L=21.435$. At this size the traveling impulse after reflection from the right boundary and next from the left boundary generates a spatially periodic stationary structure 
continuously. Therefore, one may expect that the source of traveling impulses decaying before the left boundary may appear periodically if the size of the system is increased by the distance between two subsequent decaying pulses. These expectations are confirmed in our numerical calculations. The wavelength between subsequent sources differing by one decaying pulse is denoted by $X$ and the subinterval of $X$ on which the creation of a source of decaying impulses occurs is denoted by $\Delta x$. Sources of impulses decaying before the left boundary exist for each $L \in[14.9+n X, 14.9+\Delta x+n X](n=0,1,2, \ldots)$. The period $X$ is close to 3.11 , whereas $\Delta x$ is close to 0.21 for $B=0.42$ and $D=3.065$. The period $X$ and the subinterval $\Delta x$ do not change substantially with $L$. Sources of traveling impulses decaying before the left boundary exist for $B \in[0.418,0.422]$ and for the decreasing range of $D$ from $D \in(3.0,3.12]$ for $L=15.0$ to $D \in[3.066,3069]$ for $L=198.5$.

The selection of either the first or the second asymptotic pattern depends on the details of the spatial distributions of $s$ and $p$ when the traveling impulse approaches the left boundary. The explanation of this phenomenon is similar to that presented in our previous paper in which periodic survival or decay has been described [34]. It is based on a qualitative analysis of the competition between diffusion and kinetic terms around the front and the back of the traveling impulse, and around a local minimum between them in the concentration profile for the activator when the front approaches the impermeable boundary. Signs of the diffusion terms are well defined in these regions. The competition may affect further evolution of the traveling impulse in a qualitatively different way depending on the actual curvature of its shape. When the front approaches the left boundary, its shape changes slightly, but its velocity decreases to zero. The front velocity is determined by the threshold for excitation. The threshold depends on the actual value of the inhibitor concentration $(p)$ before the front. For Eqs. 11 and 12, the threshold increases when the value of $p$ before the front decreases.

If the traveling impulse of the activator, $s(x, t)$, reaches the boundary at the very beginning of its division, its continuation is prevented. The positive diffusion term in the region of initiation of the division (the local minimum between the front and the back) is able to prevail over the negative kinetic term for the activator in the region of initiation of the division. Moreover, the negative diffusion terms around the front of the traveling impulse, as well as around its back, are not able to prevail over the positive kinetic terms for the activator in these regions. Consequently, the division of the traveling impulse is stopped, and then reversed. After a complex evolution accompanied by changes of the front of the impulse to the back of it and vice versa, the traveling impulse survives and then starts to spread to the right boundary. The reflected impulse meets the next traveling impulse and both of them are stopped. A spatially periodic stationary structure starts to form at the left boundary.

However, if the traveling impulse reaches the boundary when its division is more advanced, then the kinetic term for the activator around the minimum between the front and the back becomes stronger and prevails over the positive diffusion term in this region, and the division is completed. Moreover, the negative diffusion terms around the pulse and the impulse are sufficiently large to prevail over the positive kinetic terms, and consequently, the pulse and next the impulse itself decay. This 
scenario is realized for the next traveling impulse generated by the pulse close to the right boundary and a source of traveling impulses decaying before the left boundary is formed.

If the traveling impulse reaches the boundary when its division is much more advanced than in the previous case, then it splits into the impulse and the pulse. The negative diffusion term around the maximum of the pulse becomes sufficiently large to prevail over the positive kinetic term and, consequently, the pulse decays. However, the kinetic term for the impulse becomes so strong that it prevails over the negative diffusion term and, consequently, the impulse survives and starts to spread to the right boundary. The reflected impulse meets the next traveling impulse and they both stop. The creation of a stationary periodical structure starts.

Sources of decaying impulses appear due to specific initial conditions. If initial excitation is positioned not at the boundary but inside the system, then two traveling impulses spread to opposite boundaries. Because the subinterval of $X$ on which spatially periodic stationary structures are asymptotic solutions is much larger than $\Delta x$ on which sustained sources of decaying impulses exist, the traveling impulses usually reflect from the boundaries and asymptotically form a spatially periodic stationary structure. Therefore, the spatially periodic stationary structures are asymptotic solutions for all sizes of the system, but at some specific sizes given by $L \in[14.9+n X, 14.9+\Delta x+n X](n=0,1,2, \ldots)$ they coexist with the sources of decaying impulses.

It is noteworthy that reaction-diffusion equations are invariant to translations in space. Moreover, they are also invariant to the change of $x$ to $(-x)$, which follows from the mirror symmetry of their solutions. Therefore, the solutions to Eqs. 11 and 12 on the interval $[-L, L]$ with the initial condition located symmetrically around $x=0$ is equal to the solution on the interval $[0, L]$ and its mirror reflection. It is evident that mirror reflections can be repeated as many times as one wants. In this way, one can create any desired number of sources of traveling impulses existing in the enlarged system obtained by a corresponding number of mirror reflections.

\section{Discussion}

The best known example of a source of traveling impulses is the target pattern observed in a bromate-bromomalonic acid-ferroine system [16]. In this system as well as in all other experimental systems and models known so far, an increase of the system size causes an increase of the number of observed traveling impulses which decay not before but at impermeable walls. To the best of our knowledge, the sources of traveling impulses appearing periodically at particular sizes of the system described above have not been mentioned in the literature. Moreover, traveling impulses generated by these sources decay before impermeable walls. The selection between asymptotic patterns, namely, traveling impulses decaying before the walls and spatially periodic stationary structures, observed at periodical increase of the size of the 1D system has been found in the model (11) and (12) [34]. The periodical selection of asymptotic patterns described in the present paper is the second 
example of the periodical dependence of asymptotic solutions on the size of 1D reaction-diffusion systems discussed in the literature.

The periodical sensitivity of patterns with traveling fronts on a system size has been observed in 2D nonlinear reaction-diffusion (RD) systems. Numerical solutions of the 2D Kuramoto-Sivashinsky equation for mixed-order autocatalytic kinetics have shown that, if the width of the system increases, the planar traveling front becomes unstable and a cellular traveling front appears [35]. A continuous increase of the system size causes instability of the cellular traveling front and the appearance of a stable non-planar oscillating traveling front followed by its instability and the appearance of a turbulent structure. Further increase of the system size causes the appearance of a new cellular front with one more cell and the sequence of bifurcations mentioned above is repeated [35]. The instability of planar traveling fronts has been observed in experiments in iodate-arsenous acid and chlorite-tetrathionate systems as well as in their models [36, 37]. Planar traveling impulses can also become unstable if the width of the 2D nonlinear RD system with reactions 1-10 increases above some critical value. A cellular traveling impulse appears which transforms into a developing stationary periodical pattern which periodically emits cellular traveling impulses [32]. A circular traveling impulse initiated in a 2D nonlinear RD model of a thermochemical system also becomes unstable and a spatio-temporal periodical pattern is generated as both sizes of the system increase above some critical value [38]. Let us also mention that patterns mimicking all capital Latin letters [30] as well as all Old Hebrew letters [31] may be obtained exclusively by changing the shape of the 2D RD system with reactions $1-10$.

It is possible that the phenomenon described in the present paper can be observed in generic models commonly used to describe traveling impulses, like the FitzHugh-Nagumo [4, 7] and the Gray-Scott [8, 12] models, provided that the qualitative assumptions characterizing our model are fulfilled. Also the $1 \mathrm{D}$ version of the model suggested for describing a surface reaction (CO oxidation on $\operatorname{Pt}(110)$ ) [39] is promising, because the qualitative change of the pattern with the size of the system might be observed experimentally, provided that the experimental setup was as close to $1 \mathrm{D}$ as possible. It is worth noting that our model is also realistic and could be realized in experiments in a 1D CFUR with two enzymatic reactions. One of them should be inhibited by its reactant and product. There are many enzymes which are inhibited by an excess of their reactants and products. Examples include invertase inhibited by sucrose (reactant) and by fructose and glucose (products), xantine oxidase inhibited by xantine (reactant) and ureate (product), acetylcholinesterase inhibited by acetylcholine and choline, and many others [40, 41]. The appearance of various spatiotemporal paterns has been reported by Sheintuch and Nekhamkina in the $1 \mathrm{D}$ cross-flow reactor [42]. The stationary structure is formed due to transition from a spatially infinite system to a bounded one.

We hope that our results may be helpful for seeking the phenomenon in real systems. It is not excluded that the target patterns can appear in the BrayLiebhavsky reaction for which the model describing all observed time patterns has been elaborated by the Belgrade group [43, 44]. 
Open Access This article is distributed under the terms of the Creative Commons Attribution 4.0 International License (http://creativecommons.org/licenses/by/4.0/), which permits unrestricted use, distribution, and reproduction in any medium, provided you give appropriate credit to the original author(s) and the source, provide a link to the Creative Commons license, and indicate if changes were made.

\section{References}

1. Zhabotinsky AM (1974) Self-oscillating concentrations. Nauka, Moscow

2. Nicolis G, Prigogine I (1977) Self-organization in nonequilibrium systems. Wiley-Intsci, New York

3. Fife PC (1979) Mathematical aspects of reacting and diffusing systems. Springer, Berlin

4. Winfree AT (1980) The geometry of biological time. Springer, New York

5. Field RJ, Burger M (1985) Oscillations and traveling waves in chemical systems. Wiley, New York

6. Vasilev VA, Romanovsky YuM, Yahno VG (1987) Autowaves processes. Nauka, Moscow (in Russian)

7. Murray JD (1989) Mathematical biology. Springer, New York

8. Gray P, Scott SK (1990) Chemical oscillations and instabilities: nonlinear chemical kinetics. Oxford University Press, Oxford

9. Kawczyński AL (1990) Chemical reactions from equilibrium through dissipative structures to chaos. WNT, Warsaw (in Polish)

10. Kerner BS, Osipov VV (1991) Autosolitons. Nauka, Moscow (in Russian)

11. Keener BS, Osipov WW (1994) Autosolitons. Kluwer, Dordrecht

12. Kapral R, Showalter K (1995) Chemical waves and patterns. Kluwer, Dordrecht

13. Orlik M (1996) Oscillating reactions. Order and Chaos, WNT, Warsaw (in Polish). ISBN 83-2041970-0

14. Murray JD (2003) Mathematical biology, II: spatial models and biomedical applications. Springer, New York

15. Vanag VK (2008) Dissipative structures in reaction-diffusion systems. Institute of Computer Researches, Moscow (in Russian)

16. Zaikin AN, Zhabotinsky AM (1970) Nature 225:525

17. Ortoleva P, Ross J (1973) J Chem Phys 58:5673

18. Ortoleva P, Ross J (1974) J Chem Phys 60:5090

19. Field RJ, Noyes RM (1974) J Am Chem Soc 96:2001-2006

20. Zhabotinsky AM, Zaikin AN (1973) J Theor Biol 40:45-61

21. Zaikin AN, Kawczyński AL (1977) J Non-Equilib Thermodyn 2:39

22. Kawczyński AL (1986) Pol J Chem 60:223-237

23. Kawczyński AL, Górski J (1981) Bull Acad Pol Sci Ser Sci Chim 29:261-268

24. Kawczyński AL, Górski J (1983) Pol J Chem 57:187-199

25. Ohta T, Hayase Y, Kobayashi R (1996) Phys Rev E 54:6074

26. Leda M, Kawczyński AL (2004) J Phys Chem A 108:7660-7670

27. Petrov V, Scott SK, Showalter K (1994) Philos Trans R Soc Lond A 347:631-642

28. Kosek J, Marek M (1995) Phys Rev Lett 74:2134-2137

29. Kawczyński AL, Legawiec B (2001) Phys Rev E 63:021405

30. Kawczyński AL, Legawiec B (2001) Phys Rev E 64:056202

31. Kawczyński AL, Legawiec B (2004) Polish J Chem 78:733

32. Kawczyński AL, Leda M, Legawiec B (2006) Phys Rev E 73:046128

33. Tikhonov AN (1952) Sb Math 31:575

34. Kawczyński AL, Legawiec B (2006) Phys Rev E 73:026112

35. Horvath D, Petrov V, Scott SK, Showalter K (1993) J Chem Phys 98:6332

36. Horvath D, Showalter K (1995) J Chem Phys 102:2471

37. Horvath D, Toth A (1998) J. Chem. Phys. 108:1447

38. Kawczyński AL, Leda M (2006) Phys Rev E 73:056208

39. Bär M, Hildebrant M, Eiswirt M, Falcke M, Engel H, Neufeld M (1994) Chaos 4:499

40. Selkov EE (1967) Oscillatory processes in biological and chemical systems. Science Publishers, Moscow 
41. Golbeter A (1996) Biochemical oscillations and cellular rhythm. Cambridge University Press, Cambridge

42. Sheintuch M, Nekhamkina O (2001) Catal Today 70:383-391

43. Vukojevic V, Anic S, Kolar-Anic L (2000) J Phys Chem A 104:10731-10739

44. Kolar-Anic L, Cupic Z, Anic S et al (1997) J Chem Soc Faraday Trans 93:2147-2152 